\title{
Induction of triacylglycerol production in Chlamydomonas reinhardtii: Comparative analysis of different element regimes
}

\author{
Zeynep E. Çakmak ${ }^{\mathrm{a}}$, Tolga T. Ölmez ${ }^{\mathrm{b}}$, Turgay Çakmak ${ }^{\mathrm{c}}$, Yusuf Menemen ${ }^{\mathrm{a}}$, Turgay Tekinay ${ }^{\mathrm{d}, \mathrm{e}, *}$ \\ ${ }^{a}$ Department of Biology, Faculty of Arts and Sciences, Kırıkkale University, 71450 Kırıkkale, Turkey \\ ${ }^{\mathrm{b}}$ UNAM, Institute of Materials Science and Nanotechnology, Bilkent University, 06800 Ankara, Turkey \\ ' Department of Molecular Biology and Genetics, Faculty of Science, Istanbul Medeniyet University, 34730 Istanbul, Turkey \\ ${ }^{\mathrm{d}}$ Gazi University, Life Sciences Application and Research Center, 06830 Ankara, Turkey

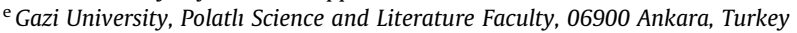

\section{H I G H L I G H T S}

- All element regimes, except S deprivation, were associated with decrease in total biovolume.

- Macro- and microelement composition of $C$. reinhardtii greatly differed.

- Overall TAG output under $\mathrm{N}, \mathrm{Mg}, \mathrm{S}$ or P deprivation was most pronounced.

- FAME profiles of N, S and P deprived cells reflect the requirements of EN 14214.

- Dramatic morphological changes were observed under different element regimes.

\section{A R T I C L E I N F O}

\section{Article history:}

Received 15 October 2013

Received in revised form 16 December 2013

Accepted 21 December 2013

Available online 31 December 2013

\section{Keywords:}

Chlamydomonas reinhardtii

Ionome

Nutrient regime

Neutral lipid

Triacylglycerol

\begin{abstract}
A B S T R A C T
In this study, impacts of different element absence (nitrogen, sulfur, phosphorus and magnesium) and supplementation (nitrogen and zinc) on element uptake and triacylglycerol production was followed in wild type Chlamydomonas reinhardtii CC-124 strain. Macro- and microelement composition of $C$. reinhardtii greatly differed under element regimes studied. In particular, heavy metal quotas of the microalgae increased strikingly under zinc supplementation. Growth was suppressed, cell biovolume, carbohydrate, total neutral lipid and triacylglycerol levels increased when microalgae were incubated under these element regimes. Most of the intracellular space was occupied by lipid bodies under all nutrient starvations, as observed by confocal microscopy and transmission electron micrographs. Results suggest that sulfur, magnesium and phosphorus deprivations are superior to nitrogen deprivation for the induction triacylglycerol production in C. reinhardtii. On the other hand, FAME profiles of the nitrogen, sulfur and phosphorus deprived cells were found to meet the requirements of international standards for biodiesel.
\end{abstract}

Crown Copyright $\odot 2013$ Published by Elsevier Ltd. All rights reserved.

\section{Introduction}

Recent increases in oil prices have encouraged much research into the use of alternative sources to accommodate the fuel requirements of modern civilization, and various organisms and organic wastes have been proposed as sustainable and environmentally friendly sources for the production of usable forms of energy such as biohydrogen, biodiesel and bioethanol. Among the liquid biofuels, biodiesels are typically formed from alkyl-esters

* Corresponding author at: Gazi University, Life Sciences Application and Research Center, 06830 Ankara, Turkey. Tel.: +90 312484 6270; fax: +90 312484 6271.

E-mail address: ttekinay@gazi.edu.tr (T. Tekinay). of long chain fatty acids, and transesterification with alcohols is a widely used method to convert lipid molecules into their alkyl-esters (Tsai et al., 2013). While oil crops such as soybean, jatropha and oil palm are particularly suitable for use in biodiesel production, unicellular algae have received considerable attention in the recent decade as rapidly proliferating, oil-rich biofuel sources with sufficient biomass output to fully replace petroleum-derived fuels (Chisti, 2007). Microalgae derived biodiesel is a second generation biofuel and does not require arable land for stock production, which prevents potential conflicts between biodiesel production and the cultivation of edible plants. In addition, microalgae possess several advantages over land crops, such as year-round growth and harvesting capacity, substantially higher biomass per area and lipid content maxima, and resistance to pests and pathogens that commonly infest crop plants (Chisti, 2007). 
Various microalgal species have been proposed for enhanced biodiesel production, including Scenedesmus obliquus, Nannochloropsis oculata, Chlorella sp., Neochloris oleoabundans (Breuer et al., 2012). In addition, common laboratory species are widely utilized as a platform for developing new strategies that would assist in reaching higher yields. Chlamydomonas reinhardtii is one of the most frequently used model organisms and has long been the center of physiological, biochemical and genetic studies for division Chlorophyta. As methods of gene transfer were developed fairly early for $C$. reinhardtii, several genetic studies have already been conducted to increase the biomass and biodiesel production of this species (Rosales-Mendoza et al., 2012). While C. reinhardtii is not widely regarded as the most suitable organism for biodiesel production at large scales, this species is nonetheless preferred in this field due to its ease of handling and capacity for non-photosynthetic growth (Rupprecht, 2009).

Despite having numerous advantages over crop plants, microalgae are associated with a number of technical, biological and economical hindrances that prevent algal biodiesels from effectively replacing petroleum-derived fuels (Chisti, 2007). The predominant biological problem is the low lipid contents displayed by many microalgal species. Under stress conditions, most microalgae direct their metabolism to the production of non-polar lipids, and especially TAG, which are used as storage molecules and rapidly degraded for energy production when optimal conditions arise. One of the most widely used stress conditions is nutrient starvation, which strongly induces TAG production of microalgae (Bölling and Fiehn, 2005). However, the exact mechanisms by which nutrient deficiencies direct the Chlamydomonas metabolism towards increased TAG production are not sufficiently explored. The present study aims to decipher the biochemical response patterns associated with the deficiency or over supplementation of several vital and trace elements by extensive quantitative and qualitative analyses, with emphasis on lipid biosynthesis. Effects of nitrogen deficiency on algal metabolism have previously been elucidated to great detail, and are utilized as a point of comparison for the effects of other element deficiencies and overabundances. Impacts of different element absences ( $\mathrm{N}, \mathrm{S}, \mathrm{P}$ and $\mathrm{Mg}$ ) and overabundances ( $\mathrm{N}$ and $\mathrm{Zn}$ ) on the microalgal metabolism have been followed by the evaluation of lipid quantities, time-based fluctuations in ion concentrations in the organism, alterations in synthesis pathways and changes in cell morphology and growth rates.

\section{Methods}

\subsection{Culturing conditions}

The wild type mt(-) 137c strain CC-124 was obtained from the Chlamydomonas Resource Center (www.chlamy.org). Cells were grown at $23^{\circ} \mathrm{C}$ under continuous light $\left(150 \mu\right.$ moles photons $\mathrm{m}^{-2}$ $\mathrm{s}^{-1}$ ) in liquid cultures on a rotary shaker $(120 \mathrm{rpm})$. Standard Tris-Acetate-Phosphate (TAP) medium was prepared as described previously. Starting cell densities were approximately $3 \times 10^{4}$ cells $\mathrm{ml}^{-1}$ in all groups. For $\mathrm{N}$ starvation studies, cells were centrifuged at $2500 \mathrm{rpm}$ for $3 \mathrm{~min}$ at room temperature, and the pellets were washed twice in TAP medium without N (TAP-N). The pellets were then resuspended in TAP-N medium and the cells were grown under constant light exposure on a rotary shaker. The same procedure was applied for other element manipulations. Initial $\mathrm{pH}$ values in all media were set to 7 prior to algal cell inoculation, $\mathrm{pH}$ values of the media were checked every $24 \mathrm{~h}$ and found not to deviate more than $8 \%$ from the initial throughout the 10 -day incubation period. Cell growth and size were monitored by haemocytometer cell counts using Lugol's solution (Sigma) and Image-J to perform volumetric calculations (Collins, 2007). Total cell biovo- lume was calculated using the equation " $B=C V$ ", in which $B$ is the total biovolume, $C$ is the number of cells, and $V$ is cell volume. For relative dry weight measurements, a volume of medium containing $1 \times 10^{9}$ cells was centrifuged at $3000 \mathrm{rpm}$ for $5 \mathrm{~min}$; and the pellet was air-dried for $5 \mathrm{~min}$, weighed, incubated at $80^{\circ} \mathrm{C}$ for $48 \mathrm{~h}$ and re-weighed. Cells from all experimental groups were harvested at the $1 \mathrm{st}, 3 \mathrm{rd}, 5 \mathrm{th}, 7$ th and 10 th days.

\subsection{Elemental analyses}

To determine metal content in algae and media, ICP mass spectrometry was utilized. Approximately $5 \times 10^{7}$ cells were collected for each treatment group, washed with $2 \mathrm{mM}$ EDTA twice, ashed in crucibles at $600{ }^{\circ} \mathrm{C}$ for $12 \mathrm{~h}$ and dissolved in $\mathrm{HNO}_{3}$. Samples were filtered prior to analysis. Elemental analysis was performed by an XSeries2 ICP-MS (Thermo Scientific, US-MA). All measurements were performed using a fully-quantified calibration method. Correlation coefficients were over $R^{2}=0.99$ for each element. Plasma power was set to 600 and $1400 \mathrm{~W}$ for cool and hot plasma applications, respectively. Isotopes with the fewest number of polyatomic interferences were chosen for $m / z$ detection in mass spectrometry. An internal standard solution (10 ppb $\mathrm{Bi}$ ) was used throughout the measurement period, measurements were repeated when internal standard concentration went beyond the tolerance range $( \pm 20 \%)$ or an unaccountably high deviation was observed between two reads of the same sample.

Concentrations of $\mathrm{C}, \mathrm{H}, \mathrm{N}, \mathrm{S}$ and $\mathrm{O}$ were measured using a Flash 2000 organic elemental analyzer (Thermo Scientific, US-MA). 3$4 \mathrm{mg}$ of oven-dried algal biomass was analyzed in tin capsules, vanadium pentoxide $\left(\mathrm{V}_{2} \mathrm{O}_{5}\right)$ was added as an oxidation catalyst prior to sealing to increase the reliability of measurements. BBOT (2,5-Bis[5-tert-butyl-benzoxazol-2yl] thiophene) was used as a standard for all measurements. A manufacturer-provided protocol for algae was utilized as the measurement method.

\subsection{Quantification of total protein and neutral lipids}

Frozen cell pellets were re-suspended in lysis buffer $(50 \mathrm{mM}$ Tris- $\mathrm{HCl} \mathrm{pH}$ 8.0, 2\% SDS, $10 \mathrm{mM}$ EDTA, and protease inhibitor mix), subjugated to sonication (3510E-DTH, Branson) for $1 \mathrm{~min}$ at $60 \%$ power $(\approx 7 \mathrm{~W} /$ pin $)$ and centrifuged at $13,000 \mathrm{~g}$ at $4{ }^{\circ} \mathrm{C}$. The supernatant was then used for protein determination with Bradford method.

Neutral lipid staining was performed using Nile Red as described by Elsey et al. (2007). Approximately $29.3 \times 10^{4}{\text { cells } \mathrm{ml}^{-1}}^{-}$ were stained with $22 \mu$ of $7.8 \times 10^{-5} \mathrm{M}$ Nile Red (Invitrogen) dissolved in acetone, left to incubate on a shaker for $15 \mathrm{~min}$ under darkness and washed twice. Relative fluorescence intensity of Nile Red staining was quantified on a fluorescence spectrometer (SpectraMax M5, MDS Analytical Technologies) using $490 \mathrm{~nm}$ excitation and $585 \mathrm{~nm}$ emission wavelengths. Total lipid levels were also confirmed gravimetrically.

\subsection{Fourier transform infrared spectroscopy (FTIR)}

A $1.3 \mathrm{ml}$ sample was concentrated and $30 \mu \mathrm{l}$ of which was then deposited on a 96 well silicon microplate and oven-dried for $45 \mathrm{~min}$ to form homogeneous thin films (Dean et al., 2010). FTIR spectra were recorded using a Nicolet 6700 Research FT-IR Spectrometer (Thermo Scientific). The bands were assigned to specific molecular groups on the basis of biochemical standards and published studies as previously described (Movasaghi et al., 2008). FTIR peak values were of particular interest which were attributed to ester group $(\mathrm{C}=0)$ vibration of triglycerides $\left(1744 \mathrm{~cm}^{-1}\right)$, carbohydrate bands $\left(1200-950 \mathrm{~cm}^{-1}\right)$ and amide I absorption $\left(1652 \mathrm{~cm}^{-1}\right)$. FTIR spectra levels of amide I band obtained from 
control cells did not deviate more than $12 \%$ therefore the amide I band was chosen for normalization of the FTIR spectra and ratio determination. Relative TAG and carbohydrate contents were determined by calculating the ratio of TAG and carbohydrate bands to the amide I band $\left(1652 \mathrm{~cm}^{-1}\right)$. Fold changes and standard errors were estimated by fitting a linear model for each time point and empirical Bayes smoothing was applied to the standard errors for all samples studied. All measurements were performed in triplicate. Spectra were baseline corrected using the automatic baseline correction algorithm and scaled to the amide I peak. The potential influence of differing cell concentrations on the changes in band intensities was eliminated by analyzing the information obtained on a per cell level.

\subsection{FAME quantitation}

FAME quantitation was performed as suggested by Praveenkumar et al. (2012) with some modifications. A $300 \mu$ l extraction buffer ( $\mathrm{MeOH}$ containing $2 \% \mathrm{H}_{2} \mathrm{SO}_{4} \mathrm{v} / \mathrm{v}$ ) was added on approximately $8 \mathrm{mg}$ lyophilized algal sample including $30 \mu \mathrm{g}$ nonadecanoic acid as internal standard. Samples were incubated for $2 \mathrm{~h}$ at $80^{\circ} \mathrm{C}$ and $750 \mathrm{rpm}$. After incubation, samples were cooled and then $300 \mu \mathrm{l}$ $\mathrm{NaCl}$ and $300 \mu \mathrm{l}$ hexane were added. After centrifugation, upper hexane layer was transferred into a glass insert vial and used for GC-MS (Agilent, 5975B) analysis. One microliter of each sample was injected into FAMEWAX column (Restek, USA) (30 m $\times 32 \mathrm{~mm}$ ID $\times 25 \mu \mathrm{m}$ film thickness). The temperature program was as follows: initial $120^{\circ} \mathrm{C}$ with $10 \mathrm{~min}$ hold; $5^{\circ} \mathrm{C}$ increment up to $230^{\circ} \mathrm{C}$ with a $5 \mathrm{~min}$ hold. Column flow was set at $22.2 \mathrm{ml} / \mathrm{min}$. The instrument condition was as follows: carrier gas nitrogen; FID set at 260, and split ratio of 10:1. Each sample was analyzed in triplicates, and FAME identification was done by comparison with standard certificate, Supelco FAME mix C4-C24 (Bellefonte, PA, USA).

\subsection{Imaging}

Confocal imaging of live cells was achieved as follows. Cells were stained with Nile Red $(5 \mu \mathrm{g} / \mathrm{ml}$ final concentration; Invitrogen). Images were acquired using an LSM 510 confocal microscope (Carl Zeiss) and a Plan Apo 63 oil immersion objective lens with a numerical aperture of 1.40-0.60. The Nile Red signal was captured using a laser excitation line at $488 \mathrm{~nm}$, and the emission was collected between 560 and $600 \mathrm{~nm}$. Chlorophyll fluorescence was captured using a laser excitation line at $633 \mathrm{~nm}$, and the emission was collected at $650 \mathrm{~nm}$. Images were merged and pseudo-colored.

TEM imaging was performed as follows. Chemicals for TEM imaging were obtained from Electron Microscopy Sciences (PA, US). $10-15 \mathrm{mg}$ of algal pellet was suspended in $1 \%$ paraformaldehyde-1\% glutaraldehyde in sodium phosphate buffer $\left(\mathrm{Na}_{2} \mathrm{HPO}_{4}-\right.$ $\mathrm{NaH}_{2} \mathrm{PO}_{4}, 1 \mathrm{M}, \mathrm{pH}=7$ ) and incubated for $24 \mathrm{~h}$. Samples were then washed with $0.1 \mathrm{M}$ sodium phosphate buffer, stained with $1 \%$ of osmium tetroxide and incubated at $4{ }^{\circ} \mathrm{C}$. Excess $\mathrm{OsO}_{4}$ was rinsed with deionized water and the samples were dehydrated in a series of alcohol solutions (70\%, 80\%, 90\%, 96\% and 100\% EtOH). Samples were then infiltrated with a $1: 1$ solution of $100 \%$ ethanol and embedding mixture (EMBED-812 resin), followed by $100 \%$ embedding mixture. Samples were placed in embedding molds and polymerized at $65^{\circ} \mathrm{C}$ for $48 \mathrm{~h}$. Ultrathin $(70-80 \mathrm{~nm})$ slices were taken by an ultramicrotome, and stained with lead citrate and uranyl acetate. Images were taken by a FEI Tecnai $\mathrm{G}^{2}$ Spirit BioTwin CTEM with average electron energy of $80 \mathrm{KeV}$.

Final data in this article are the mean values of at least three separate samples collected at two different times $(n=6)$. Means of averages are presented throughout the manuscript, and stan- dard errors and $t$-tests (two tails, pair type) with the significance criteria of $0.05,0.01$, or 0.001 are utilized to assess significance.

\section{Results and discussion}

\subsection{Growth and total lipid production of C. reinhardtii under different element regimes}

All element regimes, except $S$ deprivation, were associated with decreases in total biovolume.

In this study, $C$. reinhardtii CC- 124 cells were grown for 10 days in $\mathrm{N}, \mathrm{S}, \mathrm{P}, \mathrm{Mg}, \mathrm{K}, \mathrm{Ca}$, Fe or Zn deprived TAP media, or in TAP media supplemented with each of these elements in concentrations five times that of the base medium. All nutrient limitations studied, except those of $\mathrm{K}$ and $\mathrm{Zn}$ had a negative impact on growth, while only $\mathrm{Zn}$ and $\mathrm{N}$ over-supplementations elicited a significant decrease in growth rate (Suppl. Fig. 1). In addition, the suppression of growth was associated with an increase in neutral lipid content. (Suppl. Figs. 1 and 2). Among the element deprivations and abundances studied; N, S, P and Mg deprivations, as well as $\mathrm{Zn}$ and N abundances, caused considerable decreases in growth and increases in neutral lipid contents of microalgae (Suppl. Figs. 1 and 2). As such, microalgae grown in control (TAP), N-deficient (TAP-N), S-deficient (TAP-S), P-deficient (TAP-P), Mg-deficient (TAP-Mg), N-abundant $(\mathrm{TAP}+\mathrm{N})$ and $\mathrm{Zn}$-abundant $(\mathrm{TAP}+\mathrm{Zn})$ media were selected for further characterization.

Suppression of the growth was most pronounced in N-deprived microalgae, while other element regimes caused milder decreases in growth (Fig. 1a). Cell growth rapidly decreased in N-starved cells within the first $24 \mathrm{~h}$, while all other samples continued proliferating at varying rates and entered stationary phase approximately on day 5. Growth rates under $\mathrm{N}$ and $\mathrm{Zn}$ supplementations were slightly higher than in other regimes. The control group reached stationary phase with an OD750 value of around 0.96 on day 5 , while this value was 0.73 in $\mathrm{TAP}+\mathrm{N}, 0.64$ in $\mathrm{TAP}+\mathrm{Zn}, 0.61$ in TAP$\mathrm{S}, 0.34$ in TAP-P, 0.24 in TAP-Mg and 0.06 in TAP-N media (Fig. 1a). Nutrient limitation cause a steadily declining cell division rate and most microalgae divert and deposit fatty acids into TAG as there is no requirement for the synthesis of new membrane compounds (Sharma et al., 2012). Thus, increase in cell biovolume is an expected result and the increase in the volume of a microalga might refer to increased cytoplasmic lipid amounts. However, nitrogen deficiency was overall suboptimal for lipid production, since the immediate cessation of cell division greatly limited the total biomass attainable by N-deficient cultures. In this study, the initial cell volume of microalgae was measured as $76.6 \pm 5.7 \mu \mathrm{m}^{3}$ per cell on average. Increase in cellular biovolume was higher in $\mathrm{N}$-starved microalgae relative to control group; however, highest increase in cellular biovolume was reached in S-deprived cells on 5 th day of incubation with a maximum value of $183.9 \pm 17.2$ (Fig. 1b).

Total biovolume of each group was calculated by using data obtained from changes in cell density and cellular volume. As shown in Fig. 1c, only S starvation caused a considerable increase in total biovolume of microalgae in a culture. Two major hindrances of obtaining maximal biodiesel from microalgae strains are low cellular lipid content and poor density. Stress conditions promote neutral lipid concentration and cellular size while causing a decrease in cell concentration (Davis et al., 2011). Results in this study showed that relative dry weights of microalgae either decreased (under $\mathrm{N}$ and $\mathrm{S}$ deprivation) or remained unaffected, while total lipid levels increased dramatically under element limitation (Fig. 2). Increases in total lipid levels were highest in microalgae grown under $\mathrm{P}$ deprivation, while relative dry weight did not change significantly in this group. Likewise, the relative dry weight 

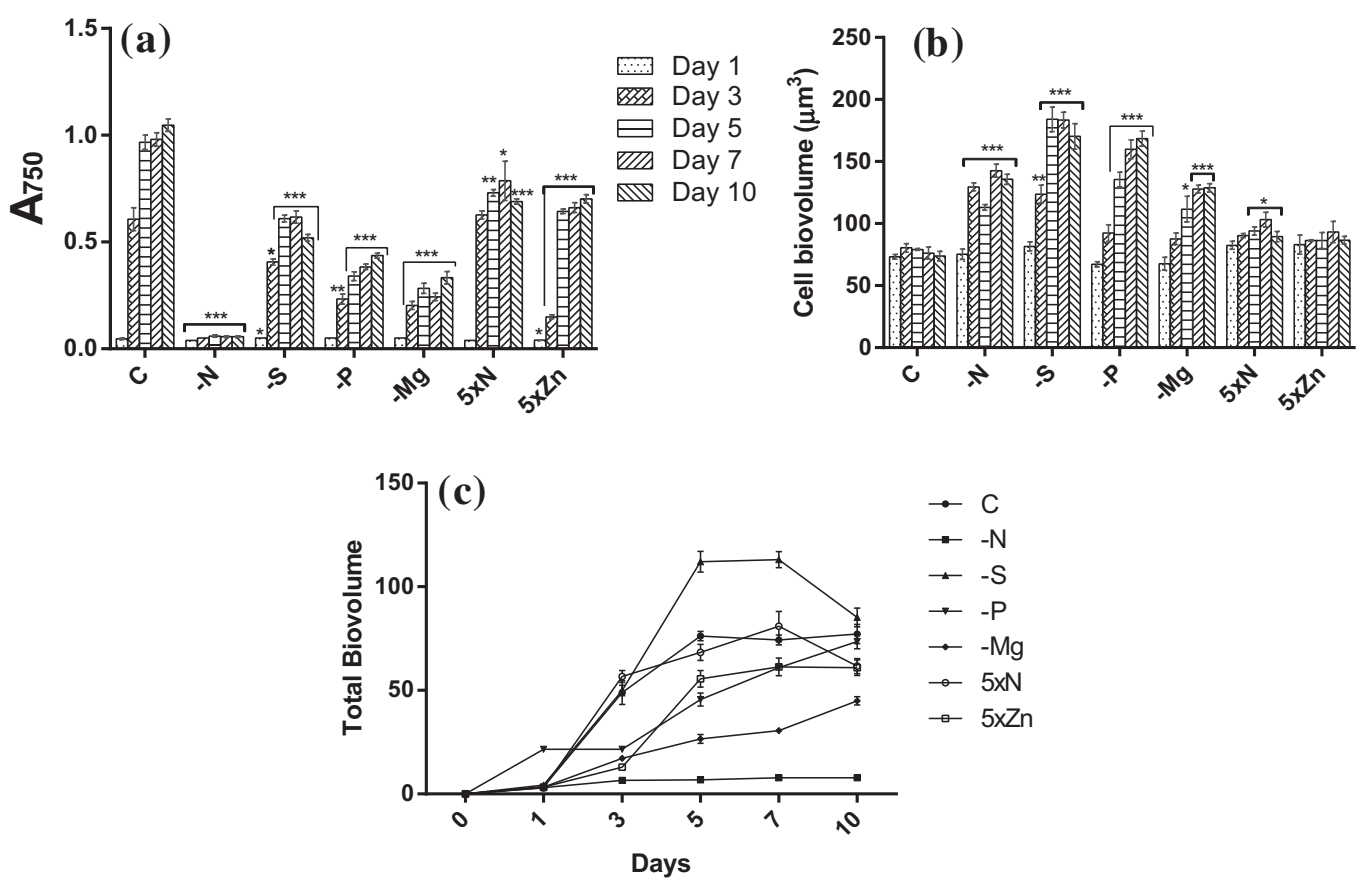

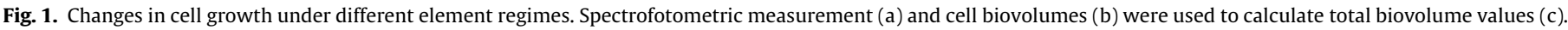

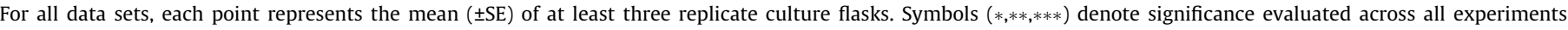
$\left(P<0.05^{*}, 0.01^{*}\right.$ or $\left.0.001^{* * *}\right)$. Significance signs were not indicated in each point to avoid complication of the Fig. 1c.

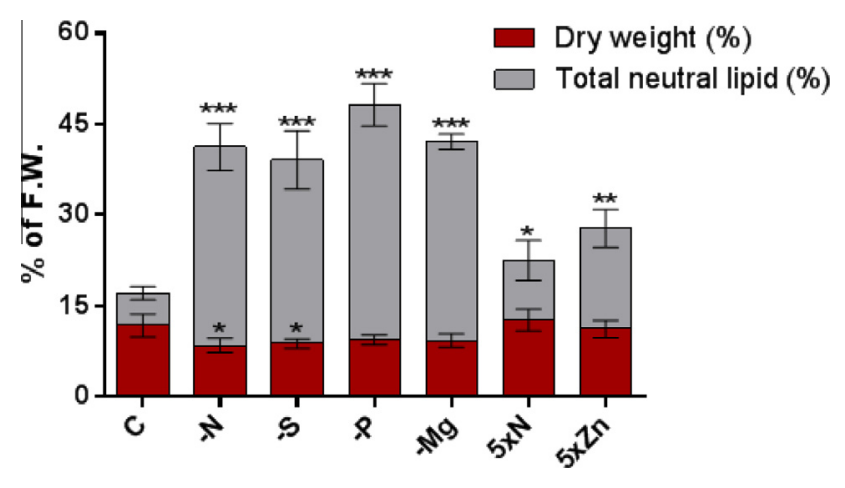

Fig. 2. Changes in dry weight and total lipid levels (on a dry weight basis) of microalgae at the end of the 10 days of incubation period under different element regimes. Approximately $1 \times 10^{9}$ cells were used for each measurement. Each data point is the mean $( \pm \mathrm{SE})$ of at least three samples. Symbols $(*, * *, * * *)$ denote significance evaluated across all experiments $\left(P<0.05^{*}, 0.01^{*}\right.$ or $\left.0.001^{* * *}\right)$.

of microalgae grown under $\mathrm{N}$ and $\mathrm{Zn}$ supplementation remained unchanged, but increase in total lipid levels were found statistically significant, albeit not as pronounced as lipid accumulations caused by nutrient deprivation (Fig. 2).

\subsection{Analysis of changes in element quotas of microalgae}

There were quite abstruse changes in element content of C. reinhardtii cells grown under $M g$ deficiency. Zinc concentration in C. reinhardtii cells increased up to 92-fold under Zn supplementation. Excess $\mathrm{Zn}$ uptake stimulated dramatic increments of other essential microelements in microalgae.

In order to evaluate element uptake efficiency of microalgae grown in described media, macro- (N, P, K, Ca, Mg and S) and micronutrient ( $\mathrm{Zn}, \mathrm{Fe}, \mathrm{Co}, \mathrm{Cu}, \mathrm{Mn}$ and $\mathrm{Mo}$ ) levels in microalgae was measured during ten days of incubation period. As expected, $\mathrm{N}, \mathrm{S}, \mathrm{P}$ and $\mathrm{Mg}$ levels of microalgae decreased by time when microalgae were grown in TAP-N TAP-S, TAP-P and TAP-Mg media respectively (Fig. 3 ). Nitrogen and $S$ content of microalgae rapidly decreased starting from the first day of incubation under $\mathrm{N}$ or $\mathrm{S}$ deprivation. It seems like presence or absence of $\mathrm{N}$ or $\mathrm{S}$ itself is a limiting factor for uptake of one of these two macroelements. On the other hand, even if there were gradual decreases starting from the first day, a rapid decrease in $\mathrm{N}$ or $\mathrm{S}$ content of microalgae was observed after 7 days of incubation under P deprivation implying that intracellular phosphorus stocks were able to compensate for the stress making phosphorus deficiency, in this perspective, not a plausible choice for obtaining rapid stress condition (Fig. 3). However, P concentration rapidly decreased starting from the first day of incubation in the absence of all macronutrients studied (Fig. 3). Results indicate that levels of three macronutrient synchronize to preserve cellular homeostasis in C. reinhardtii. Magnesium plays a major role in the production of chlorophyll, synthesis of amino acids and cell proteins, and resistance to unfavorable factors such as nutrient limitation (Finkle and Appleman, 1953). In this study, P content of microalgae decreased and $\mathrm{N}$ content even increased while $\mathrm{S}$ content of microalgae did not exhibit a significant variance under $\mathrm{Mg}$ deprivation (Fig. 3). When compared to control group, there was an increase in carbohydrate levels (Fig. 4b), total and neutral lipid levels (Figs. 2 and 5a) and the decrease in protein concentration was not as high as observed under other element deprivations studied (Fig 4a). Thus, microalgae might utilize photosynthetic energy to produce storage compounds and protein to trigger enzymatic responses for further needs rather than keeping normal metabolism which necessitates ATP production. Understanding ATP production and oxygen consumption efficiency of microalgae under Mg deficiency would provide better insight into the use of $\mathrm{Mg}$ deprivation for increasing TAG production of microalgae.

Potassium content increased in microalgae grown under all element regimes studied (Fig. 3). Potassium activates enzymes, reduces water loss, reduces respiration, preventing energy losses and builds cellulose and induce formation of starch in plants (Leigh and 

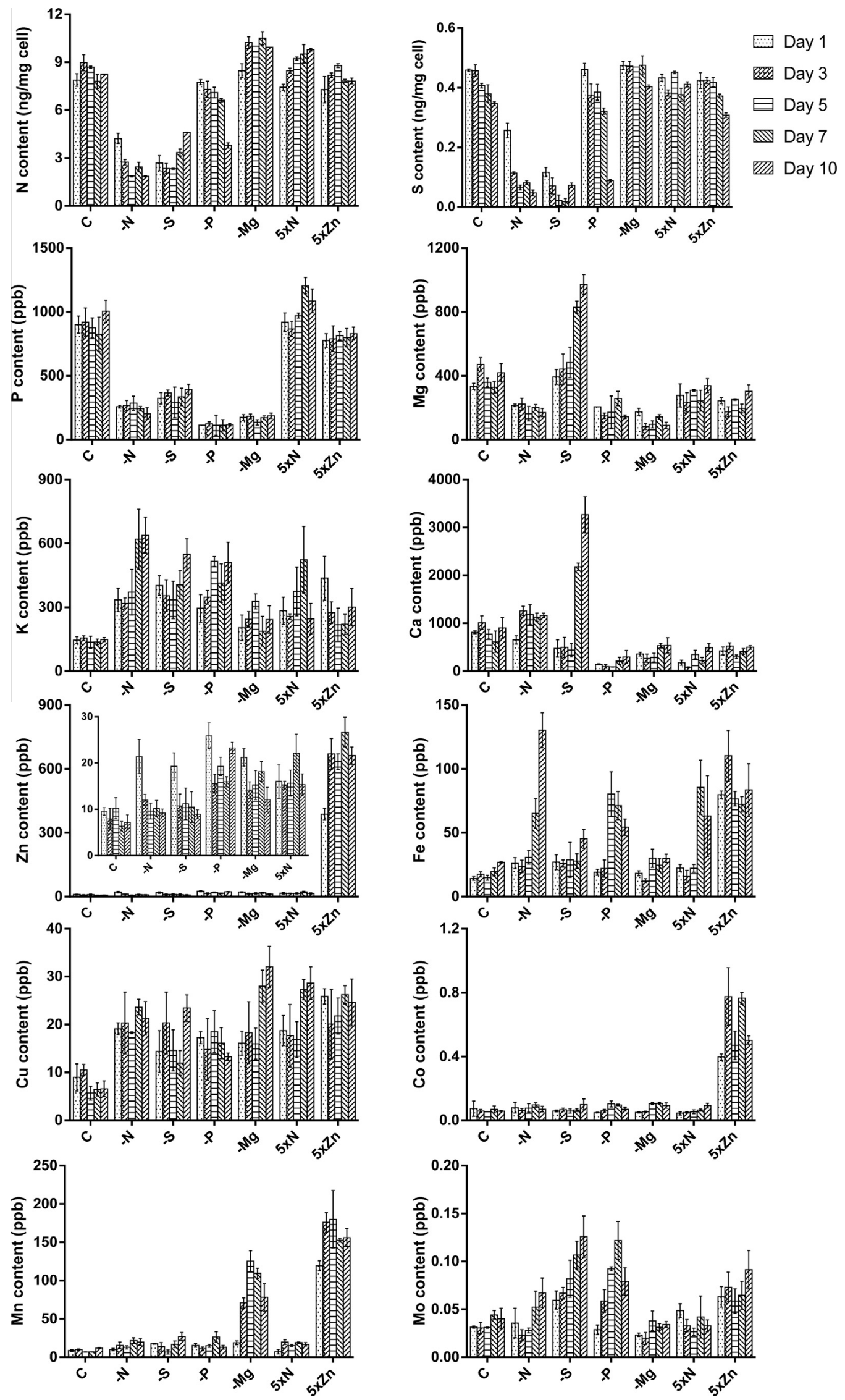

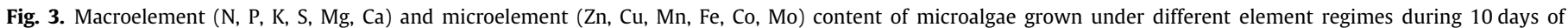
incubation. The fluctuation ranges of each point on the figures were not indicated to avoid complication of the figures. 

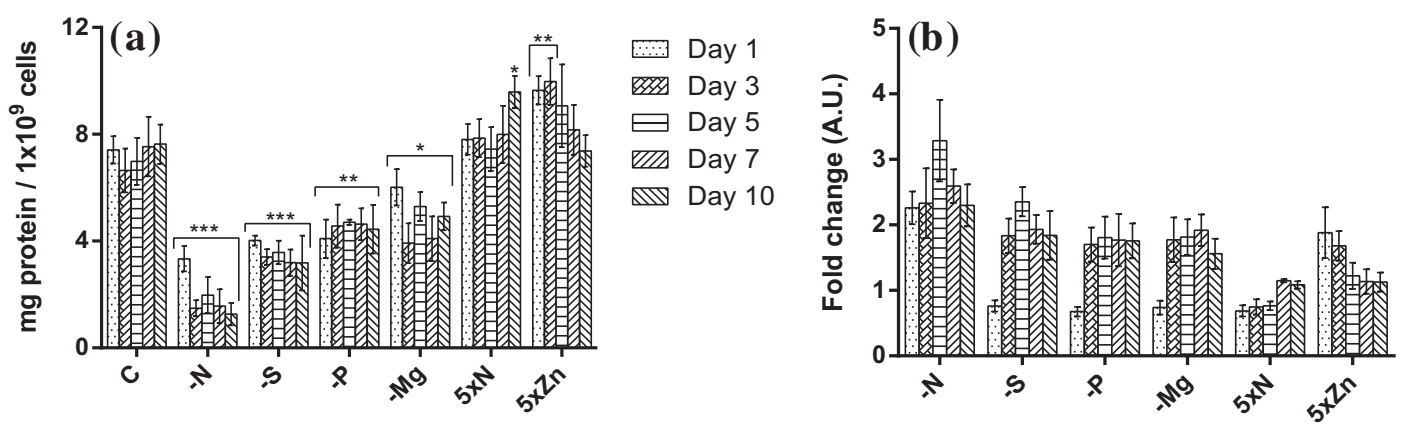

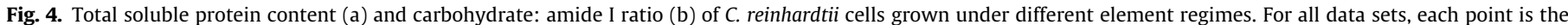

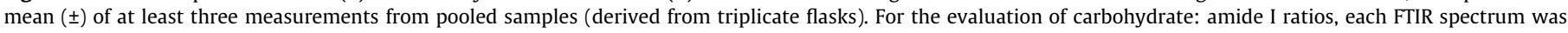

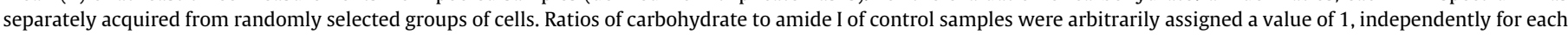
time point. Thus, comparisons of changes between different time points are not possible in this representation.
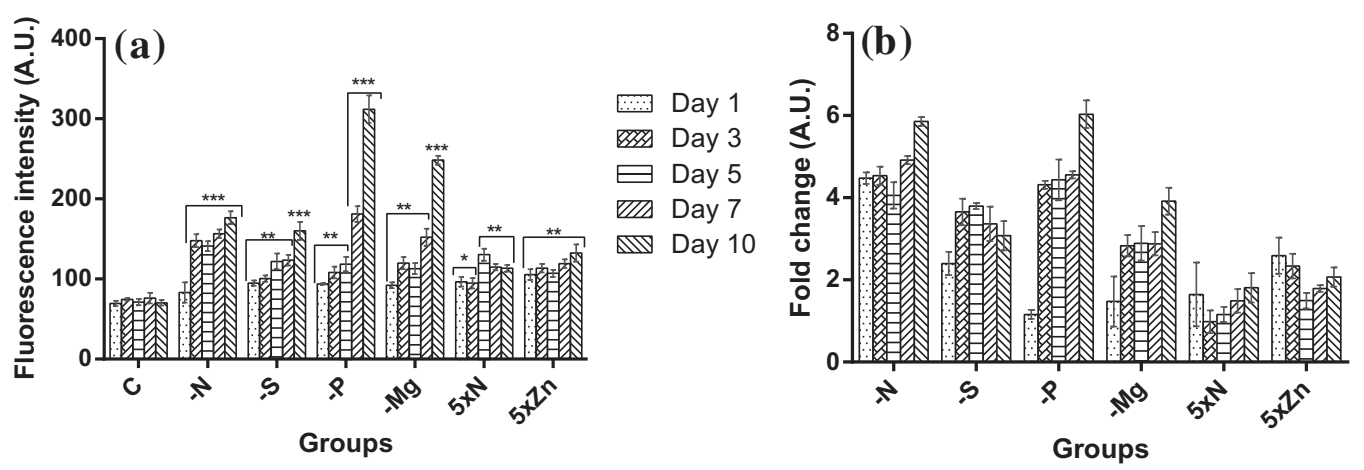

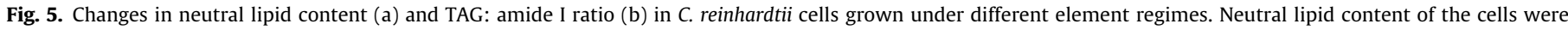

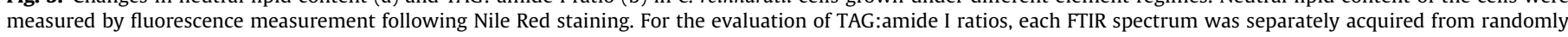

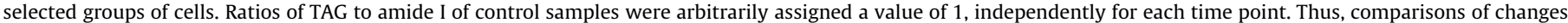
between different time points are not possible in this representation.

Jones, 1984). Increased carbohydrate formation was observed under all element regimes presented (Fig. 4b). Calcium content increased in microalgae grown in TAP-N and especially TAP-S media while significant decreases were found in microalgae grown under other element regimes. Calcium is mainly involved in determination of cell wall strength and thickness in plants (Taiz, 1984). This indicates that calcium acceptance of cell wall is enhanced under $\mathrm{N}$ and $\mathrm{S}$ deprivations that contributes to the biovolume.

Even if it would be important to understand the stoichiometry mechanisms in aqueous solution to prepare a new medium for biodiesel or high-value added products from microalgae, few studies have tested the interaction between nutrient manipulation and trace metal concentrations in microalgae. Iron limitation was reported to decrease the assimilation of nitrate and phosphate (Sterner et al., 2004), whereas similar study states an increase in Cd and Zn uptake under nitrate supplementation (Wang et al., 2001). Recently Kropat et al. (2011) offered a revised trace-element recipe for induction of TAG production from microalgae; however, the interaction between metal levels of microalgae and possible effects of the interaction of heavy metal on microalgal lipid production is in need for further detailed studies. In this study, Fe was found as the most abundant heavy metal in C. reinhardtii cells, followed by $\mathrm{Mn}, \mathrm{Zn}, \mathrm{Cu}, \mathrm{Co}$ and Mo, respectively (Fig. 3). Heavy metal concentrations of microalgae either increased or remained uneffected in response to different element regimes studied (Fig. 3). These metals play important roles as cofactor of several enzymes in plant and microalgae. Zinc content increased approximately 37-fold within the first $24 \mathrm{~h}$ and ended up with a value of 92-fold increment in TAP+Zn media in 10 days (Fig. 3). In addition, Fe, Co and $\mathrm{Mn}$ contents were higher in $\mathrm{Zn}$ supplemented microalgae from the first day and remain same (Fig. 3). Several enzymes utilize $\mathrm{Zn}$ as a cofactor, and up to $10 \%$ of all sequenced proteins from prokaryotes and eukaryotes contain Zn-binding domains (Andreini et al., 2006). Except for Mg deprived ones, Fe content of microalgae increased in all groups studied. Iron is an important substrate for the photosynthesis since the active site of glutamyl-tRNA reductase, an enzyme needed for the formation of 5-aminolevulinic acid, contains iron (Wang et al., 2001). Moreover, Fe is a cofactor of several oxidoreductases such as hydrogen dehydrogenase, superoxide dismutase and Nitrite reductases (Marschner, 1995). Thus, an increase in iron bioaccumulation within microalgae grown in supplemented media may refer to the enhanced rate of metabolism. Since growth was not enhanced in algae that are grown in supplemented media, increased iron levels may reflect activation of possible enzymatic antioxidants stemming from the increase of reactive oxygen species production. On the other hand, a boost in Fe bioaccumulation in $\mathrm{S}, \mathrm{P}$ and especially $\mathrm{N}$ deprivation may require further studies to clarify this tendency. Manganese content increased up to a maximum of 18.2-fold on 5th day and ended up with a value of 6.3-fold increase in microalgae grown under $\mathrm{Mg}$ deprivation. Manganese is an essential redox cofactor for some enzymes including some major antioxidant enzymes such as Superoxide dismutase and Catalase (Yocum and Pecoraro, 1999).

\subsection{Changes in protein, neutral lipid, TAG and carbohydrate levels}

Increases in neutral lipid, TAG and carbohydrate levels, and a corresponding decrease in protein content, were observed to be the common responses of $C$. reinhardtii strain CC-124 to element deprivation. 
Table 1

FAME composition of microalgal lipids under different element regimes. Samples were collected at the end of 10 days of incubation period.

\begin{tabular}{|c|c|c|c|c|c|c|c|c|c|}
\hline \multirow[t]{2}{*}{ Fatty acid methyl esters } & & \multirow[t]{2}{*}{ Nature } & \multicolumn{7}{|c|}{$\%$ composition under different element regimes (mean \pm SD) } \\
\hline & & & $\mathrm{C}$ & TAP-N & TAP-S & TAP-P & TAP-Mg & $\mathrm{TAP}+\mathrm{N}(5 x)$ & $\mathrm{TAP}+\mathrm{Zn}(5 x)$ \\
\hline Myristic acid & $14 ; 0$ & SFA & $0.6 \pm 0.1$ & $0.9 \pm 0.15$ & - & - & - & - & $0.2 \pm 0.03$ \\
\hline Pentadecanoic acid & $15 ; 0$ & SFA & $0.6 \pm 0.05$ & - & $1.3 \pm 0.2$ & $0.8 \pm 0.1$ & $1.8 \pm 0.3$ & $0.8 \pm 0.1$ & - \\
\hline Palmitic acid & $16 ; 0$ & SFA & $32.3 \pm 3.4$ & $43.3 \pm 1.1$ & $39.3 \pm 2.5$ & $33.3 \pm 4.1$ & $31.3 \pm 3$ & $32.7 \pm 5.1$ & $32.3 \pm 4.1$ \\
\hline Palmitoleic acid & $16 ; 2$ & PUFA & - & - & - & - & $7.2 \pm 0.8$ & $2.8 \pm 1.1$ & $1 \pm 0.2$ \\
\hline 7 Hexadesenoic acid & $16 ; 1$ & MUFA & $2.4 \pm 0.6$ & $1.7 \pm 0.4$ & $2.9 \pm 0.4$ & $4.3 \pm 1.3$ & $2.6 \pm 0.5$ & $2.9 \pm 1.2$ & $1.6 \pm 0.2$ \\
\hline 7,10-hexadecadienoic acid & $16 ; 2$ & PUFA & $0.9 \pm 0.05$ & $2.2 \pm 0.3$ & $1 \pm 0.2$ & $1.1 \pm 0.2$ & $1.1 \pm 0.1$ & $1.1 \pm 0.2$ & $3.1 \pm 0.3$ \\
\hline 7,10,13 Hexadecatrienoic acid & $16 ; 3$ & PUFA & - & - & - & - & - & $0.5 \pm 0.06$ & $1.7 \pm 0.6$ \\
\hline Hipogeic acid & $16 ; 1$ & MUFA & - & - & - & - & $1.3 \pm 0.2$ & $3.2 \pm 0.7$ & - \\
\hline Stearic acid & $18 ; 0$ & SFA & $29.2 \pm 6.4$ & $12.9 \pm 1.2$ & $33 \pm 5.8$ & $24.5 \pm 5.8$ & $15 \pm 2,1$ & $17.9 \pm 2.4$ & $16.3 \pm 3.1$ \\
\hline Lignoseric acid & $18 ; 1$ & MUFA & - & - & - & - & - & - & $3.4 \pm 0.6$ \\
\hline 7-Octadesenoic acid & $18 ; 1$ & MUFA & - & - & - & - & - & $3.3 \pm 1.1$ & - \\
\hline Oleic acid & $18 ; 1$ & MUFA & $8.3 \pm 2.1$ & $19.3 \pm 0.5$ & $11.6 \pm 2.7$ & $14.9 \pm 2.7$ & $5.2 \pm 1.2$ & $10.4 \pm 1.4$ & $9.5 \pm 2,3$ \\
\hline Linoleic acid & $18 ; 2$ & PUFA & $4.6 \pm 1.1$ & $15.4 \pm 0.2$ & $7.2 \pm 0.9$ & $9.7 \pm 1$ & $5.8 \pm 0.8$ & $6.3 \pm 1.2$ & $9.1 \pm 1.9$ \\
\hline Linolenic acid & $18 ; 3$ & PUFA & $21.1 \pm 5.4$ & $4.3 \pm 0.2$ & $3.7 \pm 1$ & $11.2 \pm 3.2$ & $26.4 \pm 4.1$ & $16.1 \pm 2.6$ & $16.8 \pm 2.8$ \\
\hline Arachidic acid & $20 ; 0$ & SFA & - & - & - & $0.2 \pm 0.01$ & - & - & - \\
\hline 11,14,17 Eicosatrienoic acid & $20 ; 3$ & PUFA & - & - & - & - & $2.3 \pm 0.4$ & $1.7 \pm 0.3$ & $1.6 \pm 0.2$ \\
\hline Eicosapentaenoic acid & $20 ; 5$ & PUFA & - & - & - & - & - & - & $3.4 \pm 0.9$ \\
\hline \multirow[t]{4}{*}{ Nevronic acid } & $24 ; 1$ & MUFA & - & - & - & - & - & $0.3 \pm 0.04$ & - \\
\hline & & $\Sigma$ SFA & $62.7 \pm 0.2$ & $57.1 \pm 0.2$ & $73.6 \pm 0.5$ & $58.8 \pm 0.3$ & $48.1 \pm 0.2$ & $51.4 \pm 0.3$ & $48.8 \pm 0.4$ \\
\hline & & $\Sigma$ MUFA & $10.7 \pm 0.1$ & $21 \pm 0.1$ & $14.5 \pm 0.2$ & $19.2 \pm 0.2$ & $9.1 \pm 0.1$ & $20.1 \pm 0.2$ & $14.5 \pm 0.2$ \\
\hline & & $\Sigma$ PUFA & $26.6 \pm 0.2$ & $21.9 \pm 0.3$ & $11.9 \pm 0.1$ & $22 \pm 0.1$ & $42.8 \pm 0.2$ & $28.5 \pm 0.4$ & $36.7 \pm 0.6$ \\
\hline
\end{tabular}

Of all nutrient starvation strategies, $\mathrm{N}$-starvation is the most widely studied approach and has been applied to a wide range of microalgal species to induce lipid production (Sharma et al., 2012). In this study, N-starved samples were used as a point of comparison for other groups studied. Element deprivation regimes generally stimulated higher increases in neutral lipid contents than $\mathrm{N}$ and $\mathrm{Zn}$ supplementations (Fig 5a). As suggested by Nile Red staining results, 10 days of $P$ deprivation leads to the highest accumulation of neutral lipid content in C. reinhardtii (Fig. 5a). This increase in the intensity of neutral lipids was recorded as $35 \%$ on the first day and increased up to $344 \%$ on 10 th day of incubation. Increases in lipid contents in response to $\mathrm{P}$ deprivation were previously reported on a number of algal species (Reitan et al., 1994). Nitrogen deprivation caused an increase of around $10 \%$ on the first day of incubation, which was followed by a sharp increase on the 3rd day of incubation (98\% over experiment initiation) and culminated in a $151 \%$ increase of neutral lipid content at the end of incubation period. Sulfur and $\mathrm{Mg}$ deprivation caused approximately $35 \%$ and $39 \%$ increase in neutral lipid content on the first day, and gradually reached up to $127 \%$ and $253 \%$ at the end of the 10 day incubation period (Fig. 5a). The rate at which the neutral lipid content increased in $\mathrm{Mg}$ and $\mathrm{S}$ deprivations was as high as in nitrogen deprivation, whereas the suppression of the growth was lower. Nitrogen and $\mathrm{Zn}$ supplementation also caused notable changes in the neutral lipid content of $C$. reinhardtii, starting with approximately $39 \%$ and $52 \%$ increments on the first day and reaching a maximum of $61 \%$ and $89 \%$ on the 10 th day (Fig. 5a). Recently Huang et al. (2013) reported that increasing the $\mathrm{N}$ concentration in the growth medium stimulates the growth and decreases the neutral lipid levels of one chlorophyte, Tetraselmis subcordiformis, and two chromophytes, N. oculata and Pavlova viridis. Results in this study do not support the data reported by Huang et al. (2013) may be due to the difference in species and the media (especially nitrogen source) used. Total lipid results obtained from Bligh and Dyer method were in concert with data obtained by Nile Red staining (Figs. 2 and 5a).

In general, element deprivations caused decreases in protein content (Fig. 4a) referring that photosynthetic energy is used more for lipid and carbohydrate storage which is validated by neutral lipid and carbohydrate measurements (Figs. 4b, 5). Decreases in protein content in various microalgae grown under N, S, P and
Mg deprivation has previously been demonstrated by several reports (Cakmak et al., 2012; Kilham et al., 1997). On the other hand, nitrogen supplementation caused an increase in protein content (Fig. 4a). However, the increase in protein content was not accompanied by an increase in growth, which implies that $C$. reinhardtii may not be regarded as a promising candidate for nitrogen removal studies.

Fourier Transform Infrared Spectroscopy (FTIR) measurement was performed to detect changes in triacylglycerol (TAG) and carbohydrate levels of microalgae grown under different element regimes. Carbohydrate (Fig. 4b) and TAG levels (Fig. 5b) increased in all element regimes when compared to respective controls. Starting with an initial increment of approximately 4.5-fold on first day, relative TAG content reached its maximum at 5.8-fold in day 10 when microalgae were grown under $\mathrm{N}$ deprivation (Fig. 5b). Under $\mathrm{S}$ deprivation, triacylglyceride level of microalgae showed 2.4-fold increases on first day and reached its maximum at 3.8-fold in day 5 and gradually decreased back to 3.1 -fold (Fig. 5b). Phosphorus and Magnesium deprivation caused similar responses. TAG content of microalgae grown in $\mathrm{N}$ and $\mathrm{Zn}$ abundant medium increased approximately 1.6 and 2.6-fold on first day and stayed similar afterwards (Fig. 5b). A considerable difference between FTIR and Nile Red fluorescence measurement of TAGs was emerged as the sensitivity of signal intensity attributed to TAGs. In this study, a 1.51-fold increase in neutral lipid levels with Nile-Red measurement was recorded while the increase was found as 5.8-fold according to FTIR band attributed to TAGs (Fig. 5a, b). Moreover, fluorescence intensity was found higher in 10-day $\mathrm{Mg}$ deprived cells than $\mathrm{N}$ deprived ones; however, FTIR results showed that $\mathrm{N}$ deprivation stimulates higher TAG production than $\mathrm{Mg}$ deprivation. Considering variation in dye absorption and fluorescence intensity and artifactual effects related calibration issues (Govender et al., 2012) FTIR measurement emerges as more reliable and precise approach compared to Nile Red staining for detection of biodiesel feedstock concentration in microalgae.

\subsection{Fatty acid composition of microalgal lipids}

FAME profiles of microalgae grown under $N, S$ and $P$ deprived conditions reflect the requirements of European biodiesel standards. 
The important biodiesel properties such as cetane number, iodine number, heat of combustion, NOx emission, oxidative stability, lubricity, viscosity and cold flow are solely dependent on the FAME profile (Francisco et al., 2010; Saraf and Thomas, 2007). Unsaturated fatty acids (UFA) in biodiesel lower cetane number and increase NOx emission. Polyunsaturated fatty acids (PUFA) are prone to oxidation which would also affect the lubricity and shelf life of biodiesel (Saraf and Thomas, 2007). Hence, biodiesel is desired to have high percent of SFA and UFA (Praveenkumar et al., 2012). In the present study, different element regimes were found to influence the fatty acid composition of $C$. reinhardtii is revealed in their FAME profiles. The SFA ratio was found higher than PUFA in all cases (Table 1). Moreover, SFA and monounsaturated fatty acid (MUFA) to PUFA ratio under most of the conditions meet the European standard EN 14214 requirements (European standard EN 14214, 2008). The SFA and MUFA content together accounted for more than $70 \%$ in most cases except for Mg deprived and $\mathrm{Zn}$ supplemented conditions (Table 1). According to EN14214, the level of C18:3 (linolenic acid) should be $\leqslant 12 \%$. When microalgae grown under $\mathrm{N}, \mathrm{S}$ or $\mathrm{P}$ deprived media, the linolenic acid contents were determined as $4.3 \%, 3.7 \%$ and $11.2 \%$ where the total PUFA ratios were found as $21.9 \%, 11.9 \%$ and $22 \%$ respectively. The FAME profiles of microalgae grown under N, S and P deprived conditions reflect the requirements of EN 14214.

\subsection{Characterization of cell morphology}

Formation of the cytoplasmic lipid bodies, loss of flagella and fragmentation of chlorophyll clusters were the most pronounced changes of microalgae grown under element deprivation.

In order to detect changes in lipid body formation and cell morphology, transmission electron microscopy (TEM) and confocal microscopy images were taken at the 10th day following nutrient manipulation (Suppl. Fig. 3). Most remarkable morphological changes were the loss of flagella, larger and spherical cell formation especially in microalgae grown under element deprivation and formation of cytoplasmic lipid droplets. Microalgae presumably responded to element deprivations and supplementations by increasing their organelle turnover rates, which resulted in the accumulation of a considerable amount of macromolecules as lipid droplets. Lipid bodies were observed to cluster and fuse into larger droplets, which could occupy most of the intracellular space. Cytoplasmic lipid droplet sizes were larger in $\mathrm{P}$ and $\mathrm{Mg}$ deprived cells than in $\mathrm{N}$ and $\mathrm{S}$ deprived cells. Lipid body formation was not widely recognized in cells grown under $\mathrm{N}$ supplemented media. However, confocal and TEM images suggest that the neutral lipid production is extensively stimulated in cells grown under $\mathrm{Zn}$ supplementation. Another noticeable change was degradation of chlorophyll clusters normally surrounding nucleus in microalgae grown under control conditions. Chlorophyll network was fragmentized extensively in $\mathrm{N}$-deprived cells while it was damaged to a lesser extent under $\mathrm{S}, \mathrm{P}$ and $\mathrm{Mg}$ deprivations and fragmentation was altogether absent under $\mathrm{N}$ and $\mathrm{Zn}$ supplementation (Suppl. Fig. 3).

\section{Conclusion}

As opposed to nitrogen starvation, growth is not suppressed entirely when microalgae are grown under S, P and Mg deprivation and chlorophyll fragmentation is not as severe as that in $\mathrm{N}$ deprivation. This study show that the lipid output of $\mathrm{P}, \mathrm{S}-$ and $\mathrm{Mg}-$ starved algae are comparable to $\mathrm{N}$-starvation case. Besides, FAME profiles of microalgae grown under $\mathrm{N}, \mathrm{S}$ and $\mathrm{P}$ deprived conditions reflect the requirement expected of biodiesel. Additionally, increases in the intracellular $\mathrm{Zn}$ levels were found to stimulate the uptake of other metals.

\section{Author contributions}

Z.E.Ç and T.T designed research and wrote the paper, Z.E.Ç, T.T.Ö, T.C and Y.M performed the research.

\section{Acknowledgements}

This study was supported by grants from the Scientific and Technological Research Council of Turkey (Project \# 112Y029), Turkish Ministry of Agriculture and Rural Affairs General Directorate of Agricultural Research (TAGEM-10/AR-GE/26) and a research fund appropriated to Kırlkkale University (BAP 2011/04). Authors thank Alper D. Özkan for technical assistance and critical reading of the manuscript.

\section{Appendix A. Supplementary data}

Supplementary data associated with this article can be found, in the online version, at http://dx.doi.org/10.1016/j.biortech.2013. 12.093 .

\section{References}

Andreini, C., Banci, L., Bertini, I., Rosato, A., 2006. Zinc through the three domains of life. J. Proteome Res. 5, 3173-3178.

Bölling, C., Fiehn, O., 2005. Metabolite profiling of Chlamydomonas reinhardtii under nutrient deprivation. Plant Physiol. 139, 1995-2005.

Breuer, G., Lamers, P.P., Martens, D.E., Draaisma, R.B., Wijffels, R.H., 2012. The impact of nitrogen starvation on the dynamics of triacylglycerol accumulation in nine microalgae strains. Bioresour. Technol. 124, 217-226.

Cakmak, T., Angun, P., Demiray, Y., Ozkan, A., Elibol, Z., Tekinay, T., 2012. Differential effects of nitrogen and sulfur deprivation on growth and biodiesel feedstock production of Chlamydomonas reinhardtii. Biotechnol. Bioeng. 109, 1947-1957.

Chisti, Y., 2007. Biodiesel from microalgae. Biotechnol. Adv. 25, 294-306.

Collins, T.J., 2007. ImageJ for microscopy. Biotechniques 43, 25-30.

Davis, R., Aden, A., Pienkos, P., 2011. Techno-economic analysis of autotrophic microalgae for fuel production. Appl. Energy 88, 3524-3531.

Dean, A.P., Sigee, D.C., Estrada, B., Pittman, J.K., 2010. Using FTIR spectroscopy for rapid determination of lipid accumulation in response to nitrogen limitation in freshwater microalgae. Bioresour. Technol. 101, 4499-4507.

Elsey, D., Jameson, D., Raleigh, B., Cooney, M.J., 2007. Fluorescent measurement of microalgal neutral lipids. J. Microbiol. Methods 68, 639-642.

European Standard N14214, 2008. Automotive Fuels - Fatty Acid Methyl Esters (FAME) for Diesel Engines - Requirements and Test Methods. European Committee for Standardization, Brussels, pp. 1-15.

Finkle, B.J., Appleman, D., 1953. The effect of magnesium concentration on growth of Chlorella. Plant Physiol. 28, 664-673.

Francisco, E.C., Neves, D.B., Jacob-Lopes, E., Franco, T.T., 2010. Microalgae as feedstock for biodiesel production: carbon dioxide sequestration, lipid production and biofuel quality. J. Chem. Technol. Biotechnol. 85, 395-403.

Govender, T., Ramanna, L., Rawat, I., Bux, F., 2012. BODIPY staining, an alternative to the Nile Red fluorescence method for the evaluation of intracellular lipids in microalgae. Bioresour. Technol. 114, 507-511.

Huang, X., Huang, Z., Wen, W., Yan, J., 2013. Effects of nitrogen supplementation of the culture medium on the growth, total lipid content and fatty acid profiles of three microalgae (Tetraselmis subcordiformis, Nannochloropsis oculata and Pavlova viridis). J. Appl. Phycol. 25, 129-137.

Kilham, S., Kreeger, D., Goulden, C., Lynn, S., 1997. Effects of nutrient limitation on biochemical constituents of Ankistrodesmus falcatus. Freshwater Biol. 38, 591596.

Kropat, J., Hong-Hermesdorf, A., Casero, D., Ent, P., Castruita, M., Pellegrini, M., Merchant, S., Malasarn, D., 2011. A revised mineral nutrient supplement increases biomass and growth rate in Chlamydomonas reinhardtii. Plant J. 66 $770-780$.

Leigh, R.A., Jones, R.G., 1984. A hypothesis relating critical potassium concentrations for growth to the distribution and functions of this ion in the plant cell. New Phytol. 97, 1-13.

Marschner, H., 1995. Mineral Nutrition in Higher Plants, second ed. Academic Publisher, London.

Movasaghi, Z., Rehman, S., Rehman, I.U., 2008. Fourier transform infrared (FTIR) spectroscopy of biological tissues. Appl. Spectrosc. Rev, 43, 134-179.

Praveenkumar, R., Shameera, K., Mahalakshmi, G., Akbarsha, M.A., Thajuddin, N., 2012. Influence of nutrient deprivations on lipid accumulation in a dominant indigenous microalga Chlorella sp., BUM11008: evaluation for biodiese production. Biomass Bioenergy 37, 60-66.

Reitan, K., Rainuzzo, J., Olsen, Y., 1994. Effect of nutrient limitation on fatty-acid and lipid-content of marine microalgae. J. Phycol. 30, 972-979. 
Rosales-Mendoza, S., Paz-Maldonado, L.M., Soria-Guerra, R.E., 2012. Chlamydomonas reinhardtii as a viable platform for the production of recombinant proteins: current status and perspectives. Plant Cell Rep. 31, 479-494.

Rupprecht, J., 2009. From systems biology to fuel - Chlamydomonas reinhardtii as a model for a systems biology approach to improve biohydrogen production. J. Biotechnol. 142, 10-20.

Saraf, S., Thomas, B., 2007. Influence of feedstock and process chemistry on biodiesel quality. Process Saf. Environ. 85, 360-364.

Sharma, K., Schuhmann, H., Schenk, P., 2012. High lipid induction in microalgae for biodiesel production. Energies 5, 1532-1553.

Sterner, R., Smutka, T., McKay, R., Qin, X., Brown, E., Sherrell, R., 2004. Phosphorus and trace metal limitation of algae and bacteria in Lake Superior. Limnol. Oceanogr. 49, 495-507.
Taiz, L., 1984. Plant-cell expansion - regulation of cell wall mechanical properties. Annu. Rev. Plant Phys. 35, 585-657.

Tsai, Y.T., Lin, H.M., Lee, M.J., 2013. Biodiesel production with continuous supercritical process: non-catalytic transesterification and esterification with or without carbon dioxide. Bioresour. Technol. 145, 362-369.

Wang, W., Dei, R., Xu, Y., 2001. Responses of Zn assimilation by coastal plankton to macronutrients. Limnol. Oceanogr. 46, 1524-1534.

Yocum, C. Pecoraro, V., 1999. Recent advances in the understanding of the biological chemistry of manganese. Curr. Opin. Chem. Biol. 3, 182-187. 